\title{
Effect of cerium additive and secondary phase analysis on $\mathrm{Ag}_{0.5} \mathrm{Bi}_{0.5} \mathrm{TiO}_{3}$ ceramics
}

\author{
S SUPRIYA* D, ANTONIO J DOS SANTOS-GARCÍA and F FERNÁNDEZ-MARTINEZ \\ Mechanical Engineering, Chemistry and Industrial Design Department, E.T.S.I.D.I., \\ Technical University of Madrid (UPM), Madrid 28012, Spain
}

MS received 27 August 2015; accepted 1 October 2015

\begin{abstract}
Cerium-doped silver bismuth titanate- $\mathrm{Ag}_{0.5} \mathrm{Bi}_{0.5} \mathrm{TiO}_{3}$ (ABT) ceramics have been synthesized by the high-temperature solid-state reaction method. The structure and elemental examination of the prepared ceramic was analysed by X-ray diffraction (XRD), Fourier transform infrared, scanning electron microscopy and energydispersive spectroscopy. XRD analysis showed the presence of pyrochlore structure and secondary phase when more than $5 \mathrm{~mol} \%$ cerium was added. The impact of temperature on cerium-doped silver bismuth titanate samples was analysed by differential thermal analysis and differential scanning calorimetry. Cerium doping caused the flaky morphology comparing with undoped sample. The homogeneity of all the samples was discussed in detail by diffuse reflectance spectrum. This is the first time the reflection process is analysed for the cerium-doped ABT system to the best of our knowledge.
\end{abstract}

Keywords. Phase transformation; thermal analysis; thermal properties; X-ray techniques.

\section{Introduction}

Synthesis of lead-free materials has become essential because of environmental safety. In recent times, investigation on several lead-free materials like bismuth layered ceramics is being widely carried out. Among them, $\mathrm{Ag}_{0.5} \mathrm{Bi}_{0.5} \mathrm{TiO}_{3}$ (ABT) is well known as an excellent key material for resonator and actuator applications [1]. In $\mathrm{ABO}_{3}$ system, the substitution of rare earth element in A-site is more important because of its improved optical, thermal and electrical properties. Normally, the ionic radius nearer to titanium $\left(\mathrm{Ti}^{4+}\right)$ is preferred for the substitution on B-site. However in case of compound like $\mathrm{Na}_{0.5} \mathrm{Bi}_{0.5} \mathrm{TiO}_{3}$ (NBT), the B-site substitution does not show significant changes in electrical and thermal properties. In ABT compound, as the ionic radius of cerium is close to A-site, cerium is substituted in bismuth site. It is well known that the addition of cerium dioxide $\left(\mathrm{CeO}_{2}\right)$ in lead-based ceramics like PZT would improve the optical and electrical properties [2].

Silver $(\mathrm{Ag})$ is the element which forms pyrochlore structure instead of perovskite under normal conditions. At room temperature the ABT exhibits orthorhombic crystal system (space group: Ibam) and at 573 and $673 \mathrm{~K}$ it changes into tetragonal (space group: $\mathrm{I} / \mathrm{mcm}$ ) and cubic (space group: $\mathrm{Pm}-3 \mathrm{~m})$, respectively [3]. It is necessary to study about the optical, thermal and microstructural properties of $\mathrm{ABO}_{3}$ compounds like ABT and NBT. The rare earth-doped NBT compounds can act as efficient light emitters [4]. The substitution of lanthanum $\left(\mathrm{La}^{3+}\right)$ in A-site leads to grain growth

\footnotetext{
*Author for correspondence (sciencepriya@gmail.com)
}

inhibition and improved grain density [5]. Similarly, it is expected that the knowledge about microstructure, optical and thermal properties of ABT ceramics with cerium as dopant is become more important for practical applications. Although the $\mathrm{ABO}_{3}$ material [6-8] has been investigated in terms of various dopants [9-11] the grain growth properties of pure and cerium-doped ABT has not been investigated much. Cerium-substituted ABT ceramics were prepared by the solid-state reaction method and their structural, thermal and optical properties were investigated.

\section{Experimental}

Cerium-doped silver bismuth titanate powders with a stoichiometry of $\mathrm{Ag}_{0.5} \mathrm{Bi}_{(0.5-x)} \mathrm{Ce}_{x} \mathrm{TiO}_{3}(x=0,0.05,0.10$ and 0.15-abbrevated as ABT, ABCT-1, 2 and 3, respectively) were synthesized by the solid-state reaction method. Reagent grade of metal oxides and carbonate powders of $\mathrm{Ag}_{2} \mathrm{O}_{3}, \mathrm{Bi}_{2} \mathrm{O}_{3}, \mathrm{CeO}_{2}, \mathrm{TiO}_{2}$ were selected as the raw materials. The constituent chemicals in suitable stoichimetry were thoroughly ground in agate mortar for $5 \mathrm{~h}$. The dried powders were calcined at $1000^{\circ} \mathrm{C}$ for $8 \mathrm{~h}$. The samples can be further heat treated at higher temperature, i.e., upto $1100^{\circ} \mathrm{C}$. But at this temperature, the melting and decomposition of sample occurs. It may be due to substantial loss of bismuth at elevated temperature [12]. The heat-treated powders were reground with ethanol for $5 \mathrm{~h}$. The obtained powder was uniaxially pressed into cylindrical pellets of $10 \mathrm{~mm}$ diameter and 1-2 $\mathrm{mm}$ of thickness using hydraulic press with pressure of $8 \times 10^{3} \mathrm{~kg} \mathrm{~m}^{-2}$. These pellets were sintered at $1000^{\circ} \mathrm{C}$ for $12 \mathrm{~h}$ in air atmosphere. 
$\mathrm{X}$-ray diffraction (XRD) patterns were recorded using an automated diffractometer-SIEMENS-model no. D5000 with $\mathrm{Cu} \mathrm{K} \alpha$ radiation $(=1.5418 \AA)$ in a wide range of Bragg angles $2 \theta\left(20^{\circ} \leq 2 \theta \leq 80^{\circ}\right)$ at a scanning rate of $2 \mathrm{~min}^{-1}$. The functional groups were analysed by Fourier transform infrared (FTIR) spectrometer (Perkin Elmer-Spectrum GXFTIR), for the range of wavelength from 377 to $2136 \mathrm{~cm}^{-1}$. The thermal analysis was performed with heating rate of

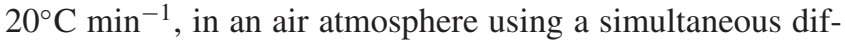
ferential thermal analysis/differential scanning calorimetry (DTA/DSC) thermal analyzer (SDT-Q600). The optical

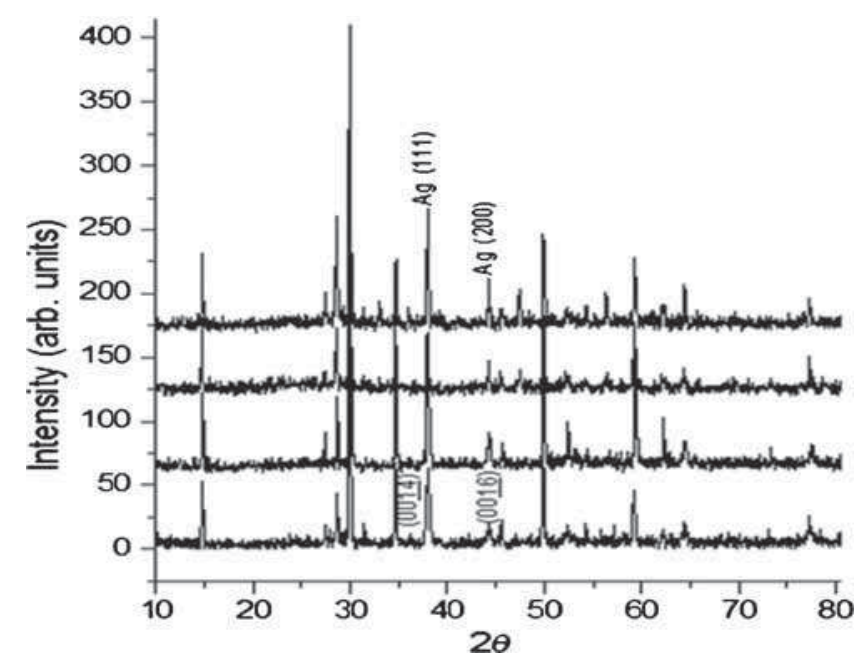

Figure 1. X-ray diffraction patterns of ceramics heat treated at $1000^{\circ} \mathrm{C}$. diffuse reflectance spectrum was obtained by reflectance spectrophotometer-Perkin Elmer-model no. Lambda-900. The morphology, microstructural features and elemental distribution of calcined powder was recorded by a scanning electron microscope (SEM) (Model no. JSM-5610 JEOL).

\section{Results and discussion}

\subsection{Powder XRD}

Figure 1 represents the XRD patterns of ABT, ABCT-1, 2 and 3 polycrystalline powder samples. The relative intensities of reflections become higher with the increase in volume of cerium oxide. It is very difficult to distinguish the $\mathrm{Ag}$ and $\mathrm{Ce}$ phase from diffraction peaks. Because the diffraction peaks of (111) and (200) metallic Ag phase locate near the cerium (0014) and (0016) reflections of bismuth titanate matrix [13]. There is no major difference in reaction phase was observed between $\mathrm{Ag}, \mathrm{Ce}$ and bismuth titanate matrix. However, in the XRD spectra, rhombohedral structure is hard to distinguish due to the overlapping of peaks. The increase in intensity at the range of $33.11^{\circ}, 47^{\circ}$ and $56.3^{\circ}(2 \theta)$ was observed. But the XRD results indicate that the cerium phase is compatible with ABT matrix. The obtained results indicate the presence of pyrochlore structure.

When cerium is doped as 10 and $15 \mathrm{~mol} \%$, the secondary phase of bismuth titanate $\left(\mathrm{Bi}_{4} \mathrm{Ti}_{3} \mathrm{O}_{12}-\mathrm{BT}\right)$ (JCPDS no. 00047-0398) has been observed at $33.5^{\circ}, 46.3^{\circ}$ and $56^{\circ}(2 \theta)$. When the \% of doping increased, the secondary BT phase dominates, and the intensity of the peaks become high. The

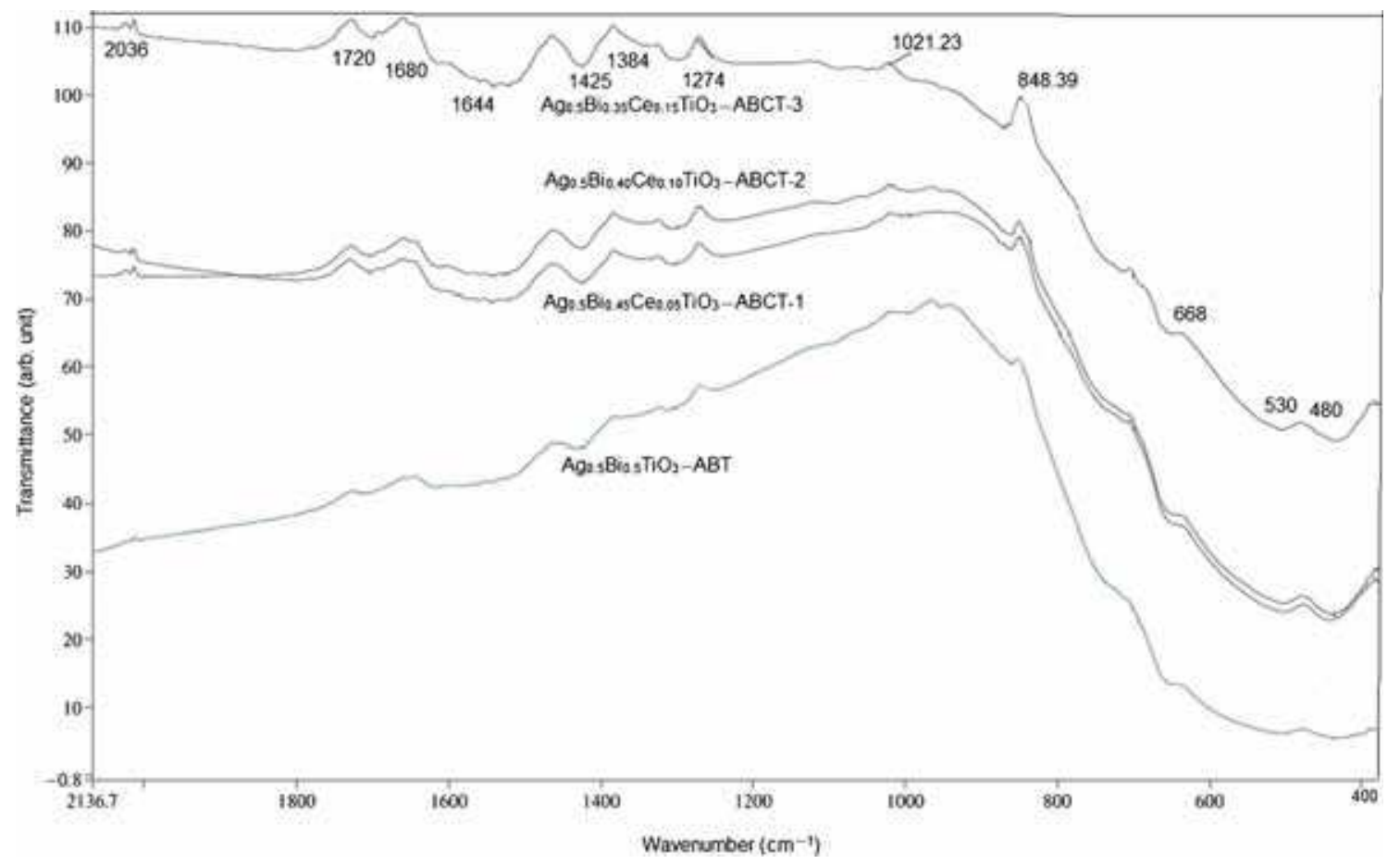

Figure 2. FTIR spectrum of ABT, ABCT-1, 2 and 3 samples. 
$\mathrm{Ce}^{3+}$ ions can be substituted in the place of $\mathrm{Bi}^{3+}$ ions. The excessive $\mathrm{Bi}^{3+}$ ions were react with $\mathrm{Ti}^{4+}$ and $\mathrm{O}^{2-}$ ions. This reaction leads to formation of secondary $\mathrm{BT}$ phase. The increase in intensity of peak with small splitting occurs at $47^{\circ}$. The compound ABT do not show any peak splitting. The doping of cerium in bismuth site leads to lattice distortion [14]. The distortion leads to splitting of peaks in ceriumdoped

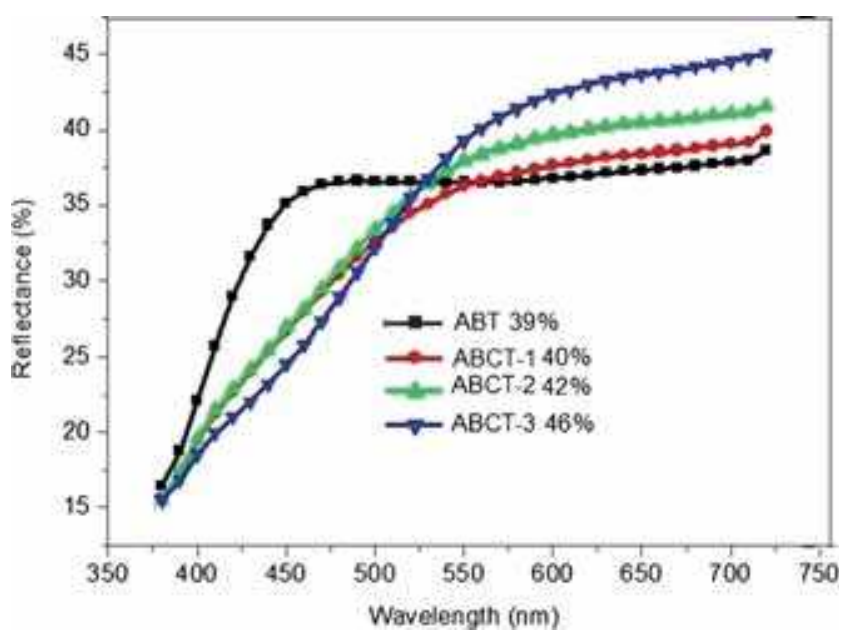

Figure 3. Reflectance spectra. samples. The peaks in all samples can be assigned as $(0$ $20 / 200)$ rhombohedral and tetragonal symmetry. The lattice parameter are calculated as [For ABCT-1, $a=3.869$ $(\AA), \alpha=90.02(\mathrm{deg})$, for ABCT-2, $a=3.887(\AA), \alpha=89.96$ (deg), for ABCT-3, $a=3.902(\AA), \alpha=90.10(\mathrm{deg})$, for ABT, $a=3.987(\AA), \alpha=90.34(\mathrm{deg})]$. The powder XRD results reveal the coexistence of rhombohedral and tetragonal phases.

\subsection{Fourier transform infrared (FTIR) spectroscopy}

FTIR spectrum of ABT, ABCT-1, 2 and 3 samples sintered at $1000^{\circ} \mathrm{C}$ are shown in figure 2 . The chemical interaction between silver $(\mathrm{Ag})$ and titanium dioxide $\left(\mathrm{TiO}_{2}\right)$ results the peak shift of the bands at 1384 and $1644 \mathrm{~cm}^{-1}$. This bands confirms the $\mathrm{Bi}-\mathrm{O}$ bonds along the $c$-axis [15]. The intensities of band between 600 and $800 \mathrm{~cm}^{-1}$ became weaker which implies the absence or lower content of organic compounds [16]. At $848.39 \mathrm{~cm}^{-1}$ the intensity of band increases as the content of cerium increases. This band confirms the presence of $\mathrm{CeO}_{2}$ in $\mathrm{ABT}$ compound [17]. The bending vibration at $480-530 \mathrm{~cm}^{-1}$ contributed to a stronger Ti-O bond [18]. The band at $668 \mathrm{~cm}^{-1}$ could be ascribed to Ti-OTi vibrations $[19,20]$. When the content of cerium increases the dramatic change in the shape of the spectra takes place between 800 and $1800 \mathrm{~cm}^{-1}$. The new peak at $1021.23 \mathrm{~cm}^{-1}$
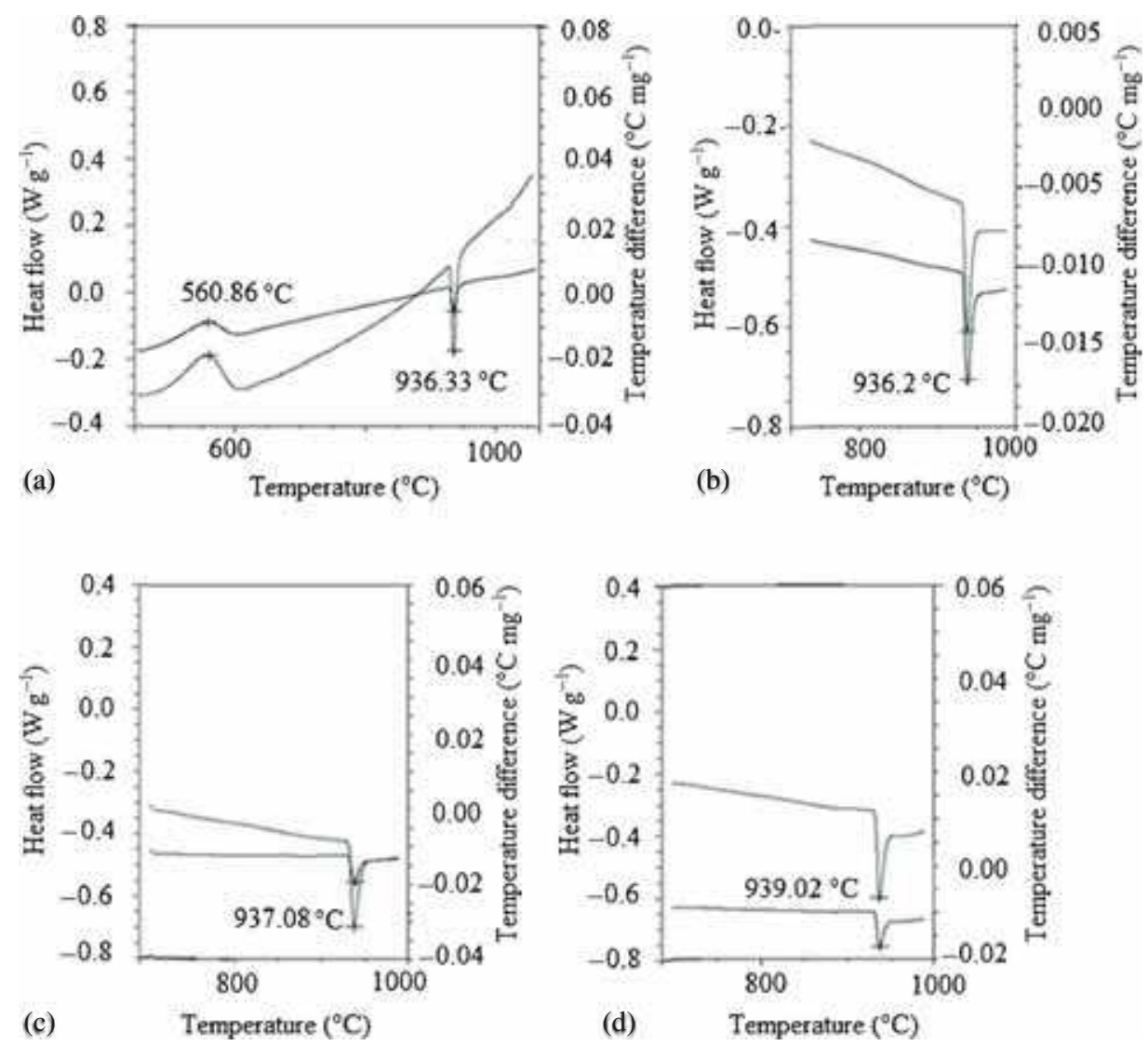

Figure 4. (a-d) Thermogravimetric analysis of ABT and ABCT samples. 
appears and the intensities of bands inceases at 2036, 1720, $1680,1650,1425$ and $1274 \mathrm{~cm}^{-1}$.

\subsection{Reflectance spectrum}

The sintered cylindrical pellets were exposed to diffuse reflectance specrum. The radiation penetrates into the samples and emerges as reflectance spectra. The values were extracted from figure 3. The samples obtained from lower to higher cerium concentration exhibit significant changes in plot, which is comparable with the ABT sample. The changes in the spectral data have been analysed with respect to the concentration of cerium. The obtained data clearly indicates the uniformity in particle size and homogeneity of the samples $[21,22]$. Colour of the samples is one of the major factors which affect the reflectance property.

The reflectance spectra illustrate the colour of the samples according to Kubelka-Munk (KM) theory. This theory explains the colour of the samples. The pellets turn from grey to pale yellow colour. This change occurs due to the presence of $3^{+}$or $4^{+}$ions of cerium and titanium. According to KM theory, the reflectance property is inversely proportional to particle size of sample. Also, this property depends upon the chemical composition of the sample and dopant of the material. The $39 \%$ of reflectance has been observed in ABT sample. However, in case of ABCT-1, 2 and 3 samples, 40, 42 and $46 \%$ of reflectance has been observed, respectively. The increase in the reflectance value is directly proportional to concentration of cerium. Based on KM theory, it is apparent that particle size decreases according to the doping $\%$ of cerium [23].

\subsection{Thermal analysis}

The thermal datas were obtained on ABT and ABCT-1, 2 and 3 samples with constant heating rate of $20^{\circ} \mathrm{C} \mathrm{min}{ }^{-1}$. The samples were heat treated upto $1000^{\circ} \mathrm{C}$ and then cooled
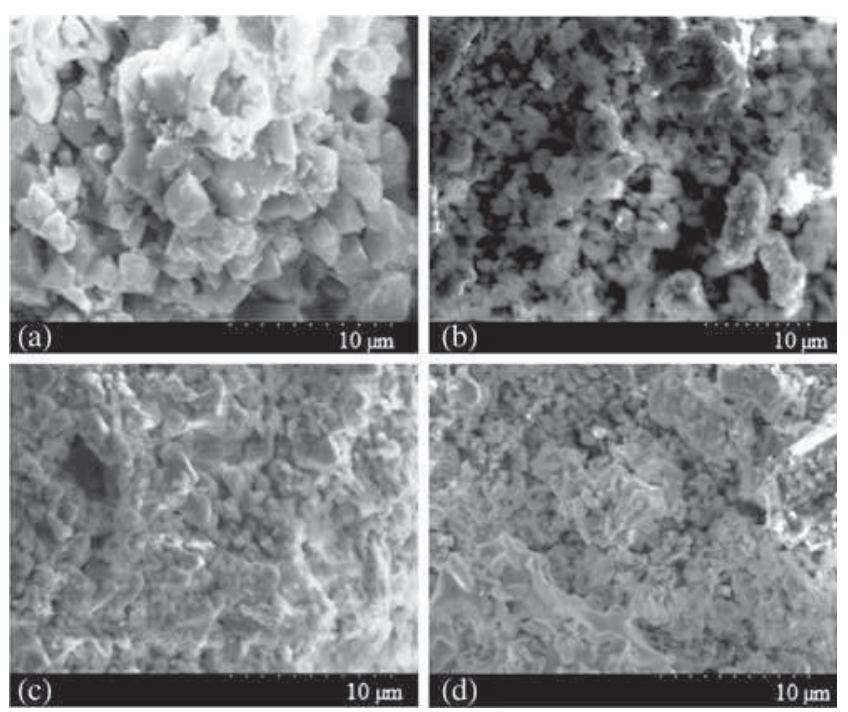

Figure 5. (a-d) SEM micrographs. down to room temperature. Figure $4 \mathrm{a}-\mathrm{d}$ illustrates DTA and DSC data of silver bismuth titanate- and cerium-doped silver bismuth titanate samples. Figure $4 \mathrm{a}$ shows the exothermic peak at $560.86^{\circ} \mathrm{C}$ and endothermic peak at $936.33^{\circ} \mathrm{C}$. Comparing XRD profiles, the endothermic peak is correlated to $\mathrm{CO}_{2}$ extraction and pyrochlore formation from the primary materials. The endothermic peaks at 936.2, 937.08, 939.02 ${ }^{\circ} \mathrm{C}$ were observed in ABCT-1, 2 and 3 samples, respectively. The difference between XRD pattern of ABT and cerium doped samples demonstrate the endothermic peak corresponds to crystallization from amorphous to pyrochlore phase transformation. The DTA/DSC and XRD results reveal that the intensity and shift in peaks are correlated to amorphous to pyrochlore phase transition process [24].

\subsection{Microstructural and elemental analysis}

Figure 5a-d shows the scanning electron microscope (SEM) image of ABT and ABCT-1, 2 and 3 samples, respectively. ABT sample forms stable cubic or spherical particles. Some plate-like particles was also appeared. This type of morphology confirms the presence of bismuth layer phase [25]. Whereas in cerium-doped samples, no cubic particles appeared. The huge number of agglomerates with flaky morphology can be seen in (b-d) samples [26]. Such morphology could be in agreement with the presence of (0014) cerium crystallites and plate-like particles confirms the (0016) reflections of bismuth titanate matrix. However, it would be very delicate to draw a definite conclusion of cerium-doped samples. Because as explained in the powder XRD, no major difference in reaction phase was observed in the $\mathrm{Ag}, \mathrm{Ce}$ and bismuth titanate combinations.

\section{Conclusion}

The present work reports the effect of incorporation of cerium in silver bismuth titanate ceramics. Increase in the intensity of XRD peak suggests the effect of cerium doping in ABT. The FTIR spectra reveals the significant changes in the $\mathrm{Bi}-\mathrm{O}$ and $\mathrm{Ti}-\mathrm{O}$ bonds. The reflectance results suggest the size of the particles at $1000^{\circ} \mathrm{C}$. The particle size decreases with respect to the doping $\%$ of cerium. The ABT sample forms the cubic structure. Whereas in cerium-doped samples the secondary phase of bismuth titanate matrix constructs the plate-like structures. Moreover, the effect of sintering temperature and cerium dopant on ABT samples was discussed in detail.

\section{Acknowledgement}

S Supriya (Erasmus mundus_-Grant no. Indi1202615) gratefully acknowledges the financial support received from India 
4EUII-European Commission Erasmus Mundus Action 2 Project (2013-2014) research fellowship.

\section{References}

[1] Gröting M and Albe K 2014 J. Solid State Chem. 213138

[2] Yueming L, Wen C, Qing X, Jing Z, Yan W and Huajun S 2007 Ceram. Int. 3395

[3] Raevski Ï I P, Reznichenko L A and Malitskaya M A 2000 Tech. Phys. Lett. 2693

[4] Rajesh Prusty K, Praveena K, Ramamurty U and Tiju T 2013 Solid State Commun. 17338

[5] Watcharapasorn A and Jiansirisomboon S 2008 Ceram. Int. 234769

[6] Ioachim A, Toacsan M I, Banciu M G, Nedelcu L, Alexandru H and Berbecaru C 2004 Mater. Sci. Eng. B 109183

[7] Herabut A and Safari A 1997 J. Am. Ceram. Soc. 802954

[8] Suchanicz J, Gavshin M G, Kudzin A Y and Kus C Z $2001 \mathrm{~J}$. Mater. Sci. Eng. B 361981

[9] Jung-Kun L, Jae Yun Y and Kug-Sun H 2001 Jpn. J. Appl. Phys. 406003

[10] Kuharuangrong S and Schulze W 1996 J. Am. Ceram. Soc. 79 1273

[11] Takenaka T, Maruyama K and Sakata K 1992 Jpn. J. Appl. Phys. 302236

[12] Khan M A, Garg A and Bell A J 2006 J. Phys. 26288
[13] Xiang P-H, Kinemuchi Y and Watari K J 2006 Electroceramics 17861

[14] Chen M, Xu Q, Kim B H, Ahn B K and Chen W 2008 Mater. Res. Bull. 431420

[15] Sun C, Wang X, Ma C and Li L 2009 J. Am. Ceram. Soc. 921613

[16] Liang Y Q, Cui Z D, Zhu S L, Liu Y and Yang X J $2011 \mathrm{~J}$. Catal. 278276

[17] Nikolina P and Vladimir V S 2009 Mater. Res. Bull. 44860

[18] Krengvirat W, Sreekantan S, Mohd Noor A-F, Chinwanitcharoen C, Muto H and Matsuda A 2012 Ceram. Int. 383001

[19] Kan Y, Wang P, Li Y, Cheng Y-B and Yan D 2002 Mater. Lett. 56910

[20] Chen D and Jiao X 2001 Mater. Res. Bull. 36355

[21] Wang L and Wang W 2012 Int. J. Hydrogen Energy 373041

[22] Ge Y, Cuikun L, Qingguo M, Stanley May P and Berry Mary T 2015 J. Lumin. 160276

[23] Sandhya Kumari L, Prabhakar Rao P, Narayana Pillai Radhakrishnan A, James V, Sameera S and Koshy P 2013 Sol. Energy Mater. Sol. Cells 112134

[24] Amini R, Ghazanfari M R, Alizadeh M, Ardakani H A and Ghaffari M 2013 Mater. Res. Bull. 48482

[25] Supriya S, Kalainathan S and Swaroop S 2011 Int. J. Chem. Tech. Res. 3488

[26] Oroosa S, Kumarb G S, Malika M M and Kurchaniaaa R 2014 Optik 125820 